\title{
Banded Matrices and Discrete Sturm-Liouville Eigenvalue Problems
}

\author{
Werner Kratz \\ Institute of Applied Analysis, University of Ulm, 89069 Ulm, Germany \\ Correspondence should be addressed to Werner Kratz, werner.kratz@uni-ulm.de
}

Received 31 August 2009; Accepted 19 November 2009

Recommended by Ondřej Dosly

We consider eigenvalue problems for self-adjoint Sturm-Liouville difference equations of any even order. It is well known that such problems with Dirichlet boundary conditions can be transformed into an algebraic eigenvalue problem for a banded, real-symmetric matrix, and vice versa. In this article it is shown that such a transform exists for general separated, self-adjoint boundary conditions also. But the main result is an explicit procedure (algorithm) for the numerical computation of this banded, real-symmetric matrix. This construction can be used for numerical purposes, since in the recent paper by Kratz and Tentler (2008) there is given a stable and superfast algorithm to compute the eigenvalues of banded, real-symmetric matrices. Hence, the Sturm-Liouville problems considered here may now be treated by this algorithm.

Copyright (c) 2009 Werner Kratz. This is an open access article distributed under the Creative Commons Attribution License, which permits unrestricted use, distribution, and reproduction in any medium, provided the original work is properly cited.

\section{Introduction}

In [1] it was shown that every discrete Sturm-Liouville eigenvalue problem (where $\Delta w_{k}=$ $\left.w_{k+1}-w_{k}\right)$

$$
L(y)_{k}:=\sum_{\mu=0}^{n}(-\Delta)^{\mu}\left\{r_{\mu}(k) \Delta^{\mu} y_{k+1-\mu}\right\}=\lambda y_{k+1} \quad \text { for } 0 \leq k \leq N
$$

with Dirichlet boundary conditions $y_{1-n}=\cdots=y_{0}=y_{N+2-n}=\cdots=y_{N+1}=0$ is equivalent with an algebraic eigenvalue problem [2] for a symmetric, banded $(N+1-n) \times(N+1-n)$ matrix with bandwidth $2 n+1$, where $N$ and $n$ are fixed integers with $1 \leq n \leq N$ (see [1, Theorem 1, Remark 1(i)]). Note that (SL) is irrelevant for $N-n+1 \leq k \leq N$ in the case of Dirichlet boundary conditions, so that in [1] equation (SL) is considered only for $0 \leq k \leq$ $N-n$. 
In this article we treat the Sturm-Liouville difference equations (SL) with general separated, self-adjoint boundary conditions. These boundary conditions include the so-called natural boundary conditions, when no or "not enough" boundary conditions are imposed [3, page 51, equation (2.3.9)]. More precisely, given (SL) and the (imposed and natural) boundary conditions, then we show that this eigenvalue problem is equivalent with an algebraic eigenvalue problem for a real-symmetric, banded matrix with bandwidth $2 n+1$, and we will construct this matrix explicity. For our general boundary conditions we must assume that the coefficients $r_{n}(k)$ are unequal to zero at "the beginning and at the end" (see (4.7) below). This leads via [4] or [5] to a numerical algorithm to compute the eigenvalues of these Sturm-Liouville eigenvalue problems.

Therefore the present paper is to some extent a continuation of the articles $[1,4]$. The paper [4] presents superfast (i.e., with $O(N)$ numerical operations) and stable algorithms for the computation of some of the eigenvalues for a real-symmetric and banded $N \times N$ matrix with bandwidth $2 n+1$, where $n=2$ or 3 for the most interesting division-free algorithms. These algorithms are based on the bisection method, and they generalize the well-known procedure for real-symmetric and tridiagonal matrices. As is shown in [1] and used in [4] the algebraic eigenvalue problems for real-symmetric, banded matrices with bandwidth $2 n+1$ are equivalent to eigenvalue problems for self-adjoint Sturm-Liouville difference equations of order $2 n$ with Dirichlet boundary conditions. Hence, these discrete Sturm-Liouville eigenvalue problems can be treated by those algorithms.

Summarizing, the main goal of this article is to provide an algorithm to calculate some of the eigenvalues of eigenvalue problems for self-adjoint Sturm-Liouville difference equations with general separated, self-adjoint boundary conditions (and not only for Dirichlet conditions as in $[4,5])$. To be more precise, we provide a construction to transform these eigenvalue problems into such eigenvalue problems with Dirichlet boundary conditions. This construction incorporates "somehow" the general boundary conditions into the first and last $2 n$ equations of the Sturm-Liouville difference equations. These transformations are stable (using essentially orthogonal transforms) and the required computational work depends on $n$ only (most interesting $n=2,3$ ) but not on $N$, so that the overall combined (with [4]) algorithm remains superfast (i.e., $\mathrm{O}(N)$ numerical operations) and stable. Hence, the results of this paper are of interest mainly for numerical applications.

Because of our intention above this article, more precisely Sections 2 to 5 of it, consists essentially of the following central parts:

(i) deriving the transform to an explicit algebraic algebraic eigenvalue problem for a symmetric, banded matrix with bandwidth $2 n+1$,

(ii) providing the required formulas, so that an implementation of the construction is easily "accessible" for the reader,

(iii) proving that the construction is always successful under the conditions (4.4) and (4.7) (which is the contents of Theorem 6.1).

This means that Sections 2 to 5 have to be quite technical. Our proceeding in these sections provides simultaneously the construction, the derivation, and the proof that the construction always works.

As already said the asserted equivalence in Theorem 6.1 is not the crucial result of this article. Actually, under the assumptions (4.4) and (4.7) this equivalence may be shown quite easily using Lemma 2.3 and Proposition 3.2. Moreover, assumption (4.4) is necessary and sufficient for self-adjointness (see, e.g., [3, Proposition 2.1.1] or elsewhere), if (4.7) holds. 
The assumption (4.7) is also necessary for all considered boundary conditions simultaneously, but for a particular boundary condition it may be weakened. But this can be seen during the procedure. The equivalence via Lemma 2.3 and Proposition 3.2 directly leads in general to a banded symmetric matrix with larger bandwidth. On the other hand the "minimal bandwidth" tridiagonal (i.e., $n=1)$ can always be achieved in a stable way by the wellknown methods of Givens and Householder, but these algorithms require $O\left(N^{2}\right)$ numerical operations as discussed in [4], which would make our whole approach obsolete.

Note that our main goal is equivalence to an "ordinary" algebraic eigenvalue problem for a real-symmetric and banded matric rather than to some matrix pencil or generalized eigenvalue problem of the form $E Y=\lambda A Y$. This is so, because the algorithm via bisection works only for these algebraic eigenvalue problems, which are well posed, while those general problems are in general not well posed. Note also that the equivalence to "some matrix pencil" can be seen immediately from (SL) or (4.1) and also (4.2). Moreover, the reduction of separated boundary conditions to Dirichlet boundary conditions via an extension of the system to a larger interval is also well known for discrete Hamiltonian or symplectic systems (see, e.g., [6] or [7]). Hence, this reduction combined with the transformation of the Sturm-Liouville equations (SL) to a linear Hamiltonian system by Lemma 2.2 would also lead to a problem with Dirichlet boundary conditions but for a larger matrix, more precisely, it would lead to some matrix pencil (possible also with larger bandwidth), which cannot be treated by the algorithms of $[4,5]$.

The discussion of assumption (4.7) in the Concluding Remarks of Section 6 does not focus on the necessity or sufficiency of it. It is quite "natural" to assume that the leading coefficient $r_{n}(k)$ never vanishes, because it is the case in most applications. But the main point is that the incorporation of our general boundary conditions into the the difference equations by our construction leads in general to problems with Dirichlet boundary conditions, but where the leading coefficient may vanish for some $k^{\prime}$ s at the beginning and the end. Therefore, algorithms via corresponding Hamiltonian or Riccati equations cannot be used anymore, so that the division-free algorithms (i.e., no divisions by $r_{n}(k)$ ) are needed as remarked in Concluding Remarks (i) of Section 6.

Let us shortly motivate why to consider the discrete Sturm-Liouville eigenvalue problems of this article, particularly for $n=2$ and $n=3$, and for general boundary conditions.

(i) The discretization of a second order Sturm-Liouville equation

$$
\left(r y^{\prime}\right)^{\prime}+q y=\lambda y
$$

of higher order leads to a banded matrix with bandwidth $2 n+1$ with $n>1$, and then even Dirichlet boundary conditions $y(a)=y(b)=0$ lead for the discrete problem to the boundary conditions $y_{0}=y_{N+1}=0$, which have to be complemented by additional "natural boundary conditions" in the usual way. Therefore, such problems of second order with Dirichlet boundary conditions cannot be treated (at least for higher-order discretization) directly by the algorithm of [4]. By using the construction of this article we obtain faster algorithms than the known ones.

(ii) Linear discretization of 4th- and 6th-order Sturm-Liouville difference equations leads to bandwidths 5 and 7, and the numerical treatment of Dirichlet boundary conditions via [4] requires also the construction of this article.

Let us shortly discuss the setup of this paper. In the next section we provide the formulae, which transform the difference equations (SL) into the corresponding matrix equation 
(see Lemma 2.1 below) based on [1]. In Section 3 we derive via partial summation the socalled Dirichlet's formula, which yields the crucial identities (3.3). In Section 4 we formulate our discrete Sturm-Liouville eigenvalue problems. In particular, we introduce (based on [8]) and discuss shortly the corresponding general separated, self-adjoint boundary conditions. In Section 5 we carry out our construction of the symmetric, banded matrix, so that the corresponding algebraic eigenvalue problem is equivalent with our Sturm-Liouville problem. Hence, our proceeding in Sections 2 to 5 provides simultaneously the construction, the derivation, and the proof that the construction always works. This is formulated as our main result in the last Section 6 by adding some concluding remarks.

\section{Discrete Sturm-Liouville Difference Equations, Banded Matrices, and Hamiltonian Systems}

Let $n \in \mathbb{N}$, and let reals $r_{\mu}(k)$ for $\mu \in\{0,1, \ldots, n\}$ and $k \in \mathbb{Z}$ be given. Then, for $y=\left(y_{k}\right)_{k \in \mathbb{Z}}$, we consider the Sturm-Liouville difference operator $L(y)$ defined by

$$
L(y)_{k}:=\sum_{\mu=0}^{n}(-\Delta)^{\mu}\left\{r_{\mu}(k) \Delta^{\mu} y_{k+1-\mu}\right\} \text { for } k \in \mathbb{Z},
$$

where $\Delta$ is the forward difference operator, that is, $\Delta w_{k}:=w_{k+1}-w_{k}$, which will always operate with respect to the variable $k$. Then, by [1, Theorem 1], we have that

$$
L(y)_{k}=(\mathscr{A} y)_{k+1} \quad \text { for } k \in \mathbb{Z}
$$

where $\mathcal{A}=\left(a_{\mu v}\right)$ is a symmetric, banded matrix with bandwidth $2 n+1$, given by

$$
\begin{aligned}
& a_{k+1, k+1+t}=(-1)^{t} \sum_{\mu=t}^{n} \sum_{v=t}^{\mu}\left(\begin{array}{c}
\mu \\
v
\end{array}\right)\left(\begin{array}{c}
\mu \\
v-t
\end{array}\right) r_{\mu}(k+v), \\
& a_{k+1, k+1-t}=(-1)^{t} \sum_{\mu=t}^{n} \sum_{v=0}^{\mu-t}\left(\begin{array}{c}
\mu \\
v
\end{array}\right)\left(\begin{array}{c}
\mu \\
v+t
\end{array}\right) r_{\mu}(k+v)
\end{aligned}
$$

for $0 \leq t \leq n$ and all $k \in \mathbb{Z}$.

This formula yields the following.

Lemma 2.1. For $k \in \mathbb{Z}$ and $y=\left(y_{v}\right)_{v \in \mathbb{Z}}$ define vectors

$$
\tilde{x}_{k}:=\left(y_{k+1-n+v}\right)_{v=0}^{n-1}, \quad v_{k}:=\left(L(y)_{k+v}\right)_{v=0}^{n-1} .
$$


Then,

$$
v_{k}=\Delta^{T}(k) \tilde{x}_{k}+M(k) \tilde{x}_{k+n}+\Delta(k+n) \tilde{x}_{k+2 n}
$$

where $M(k) \in \mathbb{R}^{n \times n}$ is a symmetric matrix, and where

$$
\Delta(k)=\left(\begin{array}{ccc}
(-1)^{n} r_{n}(k) & \cdots & 0 \\
\vdots & \ddots & \vdots \\
\star & \cdots & (-1)^{n} r_{n}(k+n-1)
\end{array}\right) \in \mathbb{R}^{n \times n}
$$

is lower triangular, and it is invertible, if $r_{n}(v) \neq 0$ for $k \leq v \leq k+n-1$. Moreover, the equation

$$
v_{k}=\lambda \tilde{x}_{k+n}
$$

is equivalent with

$$
L(y)_{k+v}=\lambda y_{k+1+v} \quad \text { for } 0 \leq v \leq n-1
$$

Next, we have by [1, Lemma 3] or [9, Remark 2] the following.

Lemma 2.2. For $k \in \mathbb{Z}$ and $y=\left(y_{v}\right)_{v \in \mathbb{Z}}$ define vectors

$$
x_{k}:=\left(\Delta^{v} y_{k-v}\right)_{v=0}^{n-1}, \quad u_{k}:=\left(\sum_{\mu=v+1}^{n}(-\Delta)^{\mu-v-1}\left\{r_{\mu}(k) \Delta^{\mu} y_{k+1-\mu}\right\}\right)_{\nu=0}^{n-1}
$$

Then, for any $k \in \mathbb{Z}$, the equation

$$
L(y)_{k+v}=\lambda y_{k+1+v}
$$

is equivalent with the Hamiltonian system

$$
\Delta x_{k}=A x_{k+1}+B_{k} u_{k}, \quad \Delta u_{k}=\left(C_{k}-\lambda \tilde{C}\right) x_{k+1}-A^{T} u_{k}
$$


provided that $r_{n}(k) \neq 0$, where one uses the following notation: $A, B_{k}, C_{k}, \tilde{C}$ are $n \times n$-matrices defined by

$$
\begin{aligned}
A:= & \left(\begin{array}{cccc}
0 & 1 & \cdots & 0 \\
\vdots & \ddots & \ddots & \vdots \\
0 & \cdots & 0 & 1 \\
0 & \cdots & 0 & 0
\end{array}\right), \quad B_{k}:=\frac{1}{r_{n}(k)} B \quad \text { with } B:=\operatorname{diag}(0, \ldots, 0,1), \\
& C_{k}:=\operatorname{diag}\left(r_{0}(k), \ldots, r_{n-1}(k)\right), \quad \tilde{C}:=\operatorname{diag}(1,0, \ldots, 0) .
\end{aligned}
$$

For the next lemma; see, for example, [10, formulae (6) and (9)] for the case of constant coefficients. It follows easily from our formulae (2.4) and (2.9) by computing the finite differences via $\Delta^{v} w_{k}=\sum_{\mu=0}^{v}\left(\begin{array}{l}v \\ \mu\end{array}\right)(-1)^{\nu-\mu} w_{k+\mu}$.

Lemma 2.3. Let $\tilde{x}_{k}$ (correspondly $\tilde{x}_{k+n}$ ), $x_{k}$, and $u_{k}$ be defined by (2.4) and (2.9). Then,

$$
\tilde{x}_{k}=T x_{k}, \quad u_{k}=T_{1}(k) \tilde{x}_{x}+T_{2}(k) \tilde{x}_{k+n},
$$

where $T=\left((-1)^{v}\left(\begin{array}{c}n-1-\mu \\ v\end{array}\right)\right)_{0 \leq \mu, v \leq n-1}$ is invertible with $T^{-1}=\left((-1)^{n-1-v}\left(\begin{array}{c}\mu \\ n-1-v\end{array}\right)\right)_{0 \leq \mu, v \leq n-1^{\prime}}$ and where $T_{1}(k), T_{2}(k)$ are $n \times n$-matrices with

$$
T_{2}(k)=\left(\begin{array}{ccc}
\star & \cdots & (-1)^{n-1} r_{n}(k+n-1) \\
\vdots & . \cdot & \vdots \\
(-1)^{0} r_{n}(k) & \cdots & 0
\end{array}\right) \text {, }
$$

and $T_{2}(k)$ is invertible, if $r_{n}(v) \neq 0$ for $k \leq v \leq k+n-1$.

\section{Dirichlet's Formula}

The next lemma is a discrete version of the continuous Dirichlet's formula [3, Lemma 8.4.3].

Lemma 3.1. For $k \in \mathbb{Z}$ and two sequences $y=\left(y_{v}\right)_{v \in \mathbb{Z}}, \bar{y}=\left(\bar{y}_{v}\right)_{v \in \mathbb{Z}}$ let the operator $L$, the vectors $x_{k}, u_{k}, \bar{x}_{k}, \bar{u}_{k}$, and the matrices $B$ and $C_{k}$ be defined as in the previous section by (2.1) and Lemma 2.2. Then, for any $k \in \mathbb{Z}$,

$$
\begin{aligned}
\bar{y}_{k+1} L(y)_{k} & =\sum_{\mu=0}^{n} r_{\mu}(k) \Delta^{\mu} \bar{y}_{k+1-\mu} \Delta^{\mu} y_{k+1-\mu}-\Delta\left(\bar{x}_{k}^{T} u_{k}\right) \\
& =\bar{x}_{k+1}^{T} C_{k} x_{k+1}+r_{n}(k)\left(\bar{x}_{k+1}-\bar{x}_{k}\right)^{T} B\left(x_{k+1}-x_{k}\right)-\Delta\left(\bar{x}_{k}^{T} u_{k}\right) .
\end{aligned}
$$


Proof. A straight forward calculation using (2.1) and (2.9) yields

$$
\begin{aligned}
\Delta\left(\bar{x}_{k}^{T} u_{k}\right)= & \bar{x}_{k+1}^{T}\left(\Delta u_{k}\right)+\left(\Delta \bar{x}_{k}^{T}\right) u_{k} \\
= & \sum_{\mu=0}^{n-1} \Delta^{\mu} \bar{y}_{k+1-\mu} \sum_{\rho=\mu+1}^{n}(-1)^{\rho-\mu-1} \Delta^{\rho-\mu}\left\{r_{\rho}(k) \Delta^{\rho} y_{k+1-\rho}\right\} \\
& +\sum_{\mu=0}^{n-1} \Delta^{\mu+1} \bar{y}_{k-\mu} \sum_{\rho=\mu+1}^{n}(-1)^{\rho-\mu-1} \Delta^{\rho-\mu-1}\left\{r_{\rho}(k) \Delta^{\rho} y_{k+1-\rho}\right\} \\
= & \sum_{\mu=1}^{n} \Delta^{\mu} \bar{y}_{k+1-\mu} r_{\mu}(k) \Delta^{\mu} y_{k+1-\mu} \\
& +\bar{y}_{k+1} \sum_{\rho=1}^{n}(-1)^{\rho-1} \Delta^{\rho}\left\{r_{\rho}(k) \Delta^{\rho} y_{k+1-\rho}\right\} \\
= & \sum_{\mu=0}^{n} r_{\mu}(k) \Delta^{\mu} \bar{y}_{k+1-\mu} \Delta^{\mu} y_{k+1-\mu}-\bar{y}_{k+1} L(y)_{k^{\prime}}
\end{aligned}
$$

which proves our assertion (3.1) using also the definition of $C_{k}$ and $B$ by Lemma 2.2.

Proposition 3.2. The matrices $T, T_{1}(k), T_{2}(k)$, and $\Delta(k)$ from Lemmas 2.3 and 2.1 satisfy

$$
T_{2}(k)=-T^{T} \Delta(k) \text { and } T_{1}(k) T \text { is symmetric. }
$$

Proof. We consider the functional $\mathscr{F}_{k}:=v_{k-n}^{T} \widetilde{\bar{x}}_{k}$, with $v_{k-n}$ and $\tilde{\bar{x}}_{k}$ defined by (2.4) of Lemma 2.1, where we put $\tilde{x}_{k-n}=0$. Then, by (2.5) of Lemma 2.1 we have that

$$
\mathcal{F}_{k}=\tilde{\bar{x}}_{k}^{T}\left\{M(k-n) \tilde{x}_{k}+\Delta(k) \tilde{x}_{k+n}\right\}
$$

From the definition of $v_{k-n}$ by (2.4) and Dirichlet's formula (3.1) we obtain that

$$
\begin{aligned}
\mathcal{F}_{k}= & \sum_{v=0}^{n-1} L(y)_{k-n+v} \bar{y}_{k-n+v+1} \\
= & \sum_{v=0}^{n-1}\left\{\bar{x}_{k-n+v+1}^{T} C_{k-n+v} x_{k-n+v+1}+r_{n}(k-n+v)\right. \\
& \left.\quad \times\left(\bar{x}_{k-n+v+1}-\bar{x}_{k-n+v}\right)^{T} B\left(x_{k-n+v+1}-x_{k-n+v}\right)\right\}-\left.\bar{x}^{T} u\right|_{k-n} ^{k} \\
= & \widetilde{\bar{x}}_{k}^{T} S \tilde{x}_{k}-\bar{x}_{k}^{T} u_{k}=(\text { by }(2.13) \text { of Lemma 2.3) } \\
= & \widetilde{\bar{x}}_{k}^{T} S \tilde{x}_{k}-\tilde{\bar{x}}_{k}^{T}\left(T^{T}\right)^{-1}\left(T_{1}(k) \tilde{x}_{k}+T_{2}(k) \tilde{x}_{k+n}\right),
\end{aligned}
$$


where $S$ is a symmetric matrix. Comparing this last formula (observe that $\tilde{\bar{x}}_{k}, \tilde{x}_{k}, \tilde{x}_{k+n}$ are completely free) with (3.4) we can conclude that

$$
-\left(T^{T}\right)^{-1} T_{2}(k)=\Delta(k) \text {, and }\left(T^{T}\right)^{-1} T_{1}(k) \text { is symmetric, }
$$

because $M(k-n)$ is symmetric by Lemma 2.1. This yields our assertion (3.3).

\section{Discrete Sturm-Liouville Eigenvalue Problems with Separated, Self-Adjoint Boundary Conditions}

Let integers $n, N \in \mathbb{N}$ with $N \geq 2 n$ (see (5.4) below) and real coefficients $r_{\mu}$ ( $k$ ) be given. Then, we consider the following discrete eigenvalue problem, which we will denote by $(\varepsilon)$. It consists of the $N+1$ self-adjoint Sturm-Liouville difference equations of even order $2 n$ (see (SL), Lemmas 2.1 and 2.2 above):

$$
L(y)_{k}=\sum_{\mu=0}^{n}(-\Delta)^{\mu}\left\{r_{\mu}(k) \Delta^{\mu} y_{k+1-\mu}\right\}=\lambda y_{k+1} \quad \text { for } 0 \leq k \leq N,
$$

and it consists of the $2 n$ linearly independent, separated, and self-adjoint boundary conditions

$$
R_{0}^{*} x_{0}+R_{0} u_{0}=0
$$

at the beginning, and

$$
R_{N+1}^{*} x_{N+1}+R_{N+1} u_{N+1}=0
$$

at the end, where $x_{0}, u_{0}, x_{N+1}, u_{N+1}$ are defined by (2.9) of Lemma 2.2, and where the real $n \times n$-matrices $R_{0}^{*}, R_{0}, R_{N+1}^{*}, R_{N+1}$ satisfy the following conditions:

$$
\operatorname{rank}\left(R_{0}^{*}, R_{0}\right)=\operatorname{rank}\left(R_{N+1}^{*}, R_{N+1}\right)=n, \quad R_{0}^{*} R_{0}^{T}, R_{N+1}^{*} R_{N+1}^{T} \text { are symmetric. }
$$

By Lemma 2.3, (4.2) and (4.3) lead to the following equivalent conditions on $\tilde{x}_{0}, \tilde{x}_{n}, \tilde{x}_{N+1}$, $\tilde{x}_{N+1+n}$, defined by (2.4), that is, on $y_{1-n}, \ldots, y_{n}$ and on $y_{N+2-n}, \ldots, y_{N+1+n}$, respectively:

$$
\begin{gathered}
R_{b}^{*} \tilde{x}_{0}+R_{b} \tilde{x}_{n}=0, \quad \text { where } R_{b}^{*}:=\left\{R_{0}^{*} T^{-1}+R_{0} T_{1}(0)\right\}, R_{b}:=R_{0} T_{2}(0), \\
R_{e}^{*} \tilde{x}_{N+1}+R_{e} \tilde{x}_{N+1+n}=0,
\end{gathered}
$$

where $R_{e}^{*}:=\left\{R_{N+1}^{*} T^{-1}+R_{N+1} T_{1}(N+1)\right\}, R_{e}:=R_{N+1} T_{2}(N+1)$, and where $T, T_{1}(\cdot), T_{2}(\cdot)$ are defined by Lemma 2.3. Moreover, the equivalence of (4.2), (4.3) with (4.5), (4.6) requires 
the assumption that $T_{2}(0)$ and $T_{2}(N+1)$ are invertible, which means by Lemma 2.3 that

$$
r_{n}(0) \cdots r_{n}(n-1) r_{n}(N+1) \cdots r_{n}(N+n) \neq 0 .
$$

We assume this from now on.

The self-adjointness of $(\mathcal{E})$ follows from general theory of linear Hamiltonian difference systems [11], and from the equivalence of our difference equation (4.1) with such systems, which is stated in Lemma 2.2. In addition, the self-adjointness of the boundary conditions via the assumption (4.4) is stated or discussed, for example, in [3, Definition 2.1.2], [8, Remark 2(iii)], [12, Proposition 2], or [7, Definition 1].

\section{Construction of the Symmetric, Banded Matrix}

First, by Lemma 2.1, the Sturm-Liouville difference equations (4.1) may be written in matrix notation, namely,

$$
A y=\lambda \tilde{y},
$$

where the coefficient matrix is of the form

$$
\mathcal{A}=\left(\begin{array}{ccccccc}
\Delta^{T}(0) & M(0) & \Delta(n) & \cdots & 0 & 0 & 0 \\
& \vdots & & \vdots & & \vdots & \\
0 & 0 & 0 & \cdots & \Delta^{T}(N+1-n) & M(N+1-n) & \Delta(N+1)
\end{array}\right)
$$

which is $\in \mathbb{R}^{(N+1) \times(N+1+2 n)}$, and where

$$
\begin{aligned}
& y=\left(y_{1-n}, \ldots, y_{N+1+n}\right)=\left(\tilde{x}_{0}^{T}, \tilde{x}_{n}^{T}, \ldots, \tilde{x}_{N+1-n}^{T}, \tilde{x}_{N+1}^{T}\right) \in \mathbb{R}^{N+1+2 n}, \\
& \tilde{y}=\left(y_{1}, \ldots, y_{N+1}\right)=\left(\tilde{x}_{n}^{T}, \ldots, \tilde{x}_{N+1-n}^{T}\right) \in \mathbb{R}^{N+1},
\end{aligned}
$$

and where we have written the first $n$ and the last $n$ rows of $\mathcal{A}$ in blocked form according to (2.4) and (2.5) of Lemma 2.1. It is the aim of this section to incorporate the boundary conditions (4.2) or (4.5) and (4.3) or (4.6) into the first $n$ and the last $n$ equations of (4.1), that is, the first and last block rows of $\mathcal{A}$, respectively. This requires in general that

$$
N \geq 2 n
$$

As a result we will obtain an algebraic eigenvalue problem for a symmetric, banded matrix of size $\left(N+1-r_{b}-r_{e}\right) \times\left(N+1-r_{b}-r_{e}\right)$ with integers $r_{b}, r_{e} \in\{0, \ldots, n\}$ depending on the boundary conditions. This algebraic eigenvalue problem will be equivalent with our given Sturm-Liouville eigenvalue problem $(\mathcal{E})$ from Section 4 under the assumptions (4.4) and (4.7). 


\subsection{Boundary Conditions at the Beginning}

We consider the boundary conditions (4.2) or (4.5) at the beginning by assuming (4.4) and (4.7). Hence, $T_{2}(0)$ and $\Delta(0)$ are invertible by Lemmas 2.1 and 2.3. Let $\tilde{X}, \tilde{U}$ be real $n \times n$ matrices such that (see (4.5))

$$
\tilde{X}^{T} \tilde{U}=\tilde{U}^{T} \tilde{X}, \quad \tilde{X}^{T} U-\tilde{U}^{T} X=I, \text { and } \tilde{X} \text { is invertible, }
$$

where $X:=-\Delta^{-1}(0)\left(R_{b}^{*}\right)^{T}, U:=R_{b}^{T}$. The existence (including construction) follows from [3, Corollary 3.3.9], because

$$
\operatorname{rank}\left(X^{T}, U^{T}\right)=n, \quad U^{T} X=X^{T} U
$$

holds. This follows from (4.4) and (4.5) by the calculations

$$
\begin{gathered}
\operatorname{rank}\left(R_{0} T_{2}(0),\left\{R_{0}^{*} T^{-1}+R_{0} T_{1}(0)\right\}\left(\Delta^{T}\right)^{-1}(0)\right)=\operatorname{rank}\left(R_{0}, R_{0}^{*}\right)=n, \\
R_{0} T_{2}(0) \Delta^{-1}(0)\left\{R_{0}^{*} T^{-1}+R_{0} T_{1}(0)\right\}^{T}=-R_{0}\left(R_{0}^{*}\right)^{T}-R_{0} T^{T} T_{1}^{T}(0) R_{0}^{T}
\end{gathered}
$$

is symmetric, where we used (3.3) of Proposition 3.2. Moreover, (C1) and (5.5) imply by [3, Proposition 1.1.5] that

$$
U \tilde{X}^{T}-\tilde{U} X^{T}=I \text {, and } X \tilde{X}^{T}, U \tilde{U}^{T} \text { are symmetric. }
$$

We conclude from (C1) and (5.7) that our boundary conditions (4.2) or (4.5) are equivalent with

$$
-\tilde{X} X^{T} \Delta^{T}(0) \tilde{x}_{0}+\tilde{X} U^{T} \tilde{x}_{n}=0
$$

Since $\tilde{X} X^{T}$ is real-symmetric by (5.7), there exists by the spectral theorem [13] an orthogonal matrix $\tilde{V}$ such that

$$
\tilde{V}^{T} \tilde{X} X^{T} \tilde{V}=\left(\begin{array}{ll}
0 & 0 \\
0 & D
\end{array}\right)
$$

where $D \in \mathbb{R}^{\left(n-r_{b}\right) \times\left(n-r_{b}\right)}$ is diagonal and invertible, so that

$$
r_{b}=n-\operatorname{rank} X=n-\operatorname{rank} R_{b}^{*}=n-\operatorname{rank}\left\{R_{0}^{*}+R_{0} T_{1}(0) T\right\} .
$$

We use this block structure from now on including the extreme cases $r_{b}=0$ and $r_{b}=n$, where the zero-matrices or $D$ do not occur. By the Gram-Schmidt process (or QR-factorization [13]), there exists an orthogonal matrix $Q \in \mathbb{R}^{\left(n-r_{b}\right) \times\left(n-r_{b}\right)}$ such that $Q^{T} \Delta_{22}$ is lower triangular, where 


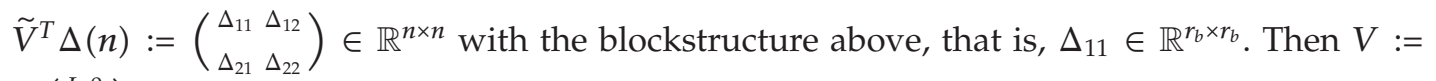
$\tilde{V}\left(\begin{array}{ll}I & 0 \\ 0 & Q\end{array}\right)$ is orthogonal, and

$$
S:=V^{T}\left(-\tilde{X} X^{T}\right) V=\left(\begin{array}{cc}
0 & 0 \\
0 & S_{22}
\end{array}\right)
$$

where $S_{22}=-Q^{T} D Q$ is symmetric and invertible. Let

$$
R:=V^{T} \tilde{X} U^{T} V=\left(\begin{array}{ll}
R_{11} & R_{12} \\
R_{21} & R_{22}
\end{array}\right)
$$

By (5.5) and (C1), $\operatorname{rank}(S, R)=n$, and

$$
S R^{T}=\left(\begin{array}{cc}
0 & 0 \\
S_{22} R_{12}^{T} & S_{22} R_{22}^{T}
\end{array}\right)=-V^{T} \tilde{X} X^{T} U \tilde{X}^{T} V
$$

is symmetric. Hence, $R_{12}=0$, because $S_{22}$ is invertible, and $\operatorname{rank}\left(\begin{array}{cccc}0 & 0 & R_{11} & 0 \\ 0 & S_{22} & R_{21} & R_{22}\end{array}\right)=n$, so that $R_{11}$ is invertible.

Altogether, we have constructed $V \in \mathbb{R}^{n \times n}$ such that

$$
S:=-V^{T} \tilde{X} X^{T} V=\left(\begin{array}{cc}
0 & 0 \\
0 & S_{22}
\end{array}\right)
$$

with a symmetric and invertible matrix $S_{22} \in \mathbb{R}^{\left(n-r_{b}\right) \times\left(n-r_{b}\right)}$, where $r_{b}=n-\operatorname{rank} X$,

$$
R:=V^{T} \tilde{X} U^{T} V=\left(\begin{array}{cc}
R_{11} & 0 \\
R_{21} & R_{22}
\end{array}\right)
$$

with an invertible matrix $R_{11} \in \mathbb{R}^{r_{b} \times r_{b}}$ and so that $S_{22} R_{22}^{T}$ is symmetric, and

$$
\tilde{\Delta}:=V^{T} \Delta(n)=\left(\begin{array}{ll}
\tilde{\Delta}_{11} & \widetilde{\Delta}_{12} \\
\widetilde{\Delta}_{21} & \widetilde{\Delta}_{22}
\end{array}\right)
$$

so that $\widetilde{\Delta}_{22} \in \mathbb{R}^{\left(n-r_{b}\right) \times\left(n-r_{b}\right)}$ is lower triangular, and where $V$ is orthogonal.

It follows immediately from (C1) and (C2) that our boundary conditions (4.2) or (4.5) are equivalent with

$$
S V^{T} \Delta^{T}(0) \tilde{x}(0)+R V^{T} \tilde{x}_{n}=0,
$$


and therefore with

$$
x_{1}(n)=0, \quad x_{2}(0)=-S_{22}^{-1} R_{22} x_{2}(n)
$$

where

$$
\tilde{\widetilde{x}}_{0}:=V^{T} \Delta^{T}(0) \tilde{x}_{0}=\left(\begin{array}{c}
x_{1}(0) \\
x_{2}(0)
\end{array}\right), \quad \tilde{\widetilde{x}}_{n}:=V^{T} \tilde{x}_{n}=\left(\begin{array}{l}
x_{1}(n) \\
x_{2}(n)
\end{array}\right)
$$

with $x_{1}(0), x_{1}(n) \in \mathbb{R}^{r_{b}}$ while $x_{1}(0)$ and $x_{2}(n)$ remain free.

We say that (5.18) is the boundary conditions in normalized form.

Now, we consider the first $n$ equations or the first block row of our difference equations (4.1) or (5.1), that is, by Lemma 2.1 and (5.2),

$$
v_{0}=\Delta^{T}(0) \tilde{x}_{0}+M(0) \tilde{x}_{n}+\Delta(n) \tilde{x}_{2 n}=\lambda \tilde{x}_{n} .
$$

We obtain from (5.7) and (5.8) that

$$
\Delta^{T}(0) \tilde{x}_{0}=\left(U \tilde{X}^{T}-\tilde{U} X^{T}\right) \Delta^{T}(0) \tilde{x}_{0}, \quad-\tilde{U} X^{T} \Delta^{T}(0) \tilde{x}_{0}=-\tilde{X} U^{T} \tilde{x}_{n}
$$

Hence, under (5.8) (i.e., the boundary conditions), (5.19) is equivalent with (use also the notation of (5.18) and (C2))

$$
\begin{aligned}
0 & =V^{T}\left(U \tilde{X}^{T} \Delta^{T}(0) \tilde{x}_{0}-\tilde{U} U^{T}\right) \tilde{x}_{n}+V^{T}\{M(0)-\lambda I\} V \tilde{x}_{n}+V^{T} \Delta(n) \tilde{x}_{2 n} \\
& =R^{T} \widetilde{\widetilde{x}}_{0}+\{\widetilde{M}(0)-\lambda I\} \widetilde{\widetilde{x}}_{n}+\widetilde{\Delta} \tilde{x}_{2 n}
\end{aligned}
$$

where $\widetilde{M}(0)=\left(\begin{array}{ll}M_{11} & M_{12} \\ M_{21} & M_{22}\end{array}\right):=V^{T}\left\{M(0)-\tilde{U} U^{T}\right\} V$ is symmetric by Lemma 2.1 and (5.7). Hence, by (C2), equation (5.19) is equivalent with (under the boundary conditions (5.18))

$$
\begin{gathered}
x_{1}(0)=-\left(R_{11}^{T}\right)^{-1}\left\{-R_{21}^{T} S_{22}^{-1} R_{22} x_{2}(n)+M_{12} x_{2}(n)+\left(\widetilde{\Delta}_{11} \tilde{\Delta}_{12}\right) \tilde{x}_{2 n}\right\}, \\
\{M-\lambda I\} x_{2}(n)+\left(\widetilde{\Delta}_{21} \widetilde{\Delta}_{22}\right) \tilde{x}_{2 n}=0, \quad \text { where } M:=-R_{22}^{T} S_{22}^{-1} R_{22}+M_{22} .
\end{gathered}
$$

Now, (5.22) defines $x_{1}(0)$ independently of $\lambda$, which was free by (5.18). Note that $M$ is symmetric, because $M(0)$ and $\tilde{U} U^{T}$ are symmetric by Lemma 2.1 and (5.7). Moreover, $\widetilde{\Delta}_{22}$ is lower triangular by (C2), so that (5.23) leads to bandwidth $2 n+1$ and symmetry. More precisely, we drop the first $n+r_{b}$ columns of $\mathcal{A}$, and the first $n$ rows are replaced by the following rows, which constitute the first $n-r_{b}$ rows of the symmetric, banded matrix under 
construction:

$$
\left(M \widetilde{\Delta}_{21} \widetilde{\Delta}_{22} 0 \cdots 0\right), \quad \text { where } M:=-R_{22}^{T} S_{22}^{-1} R_{22}+M_{22} \in \mathbb{R}^{\left(n-r_{b}\right) \times\left(n-r_{b}\right)},
$$

and where $\left(\begin{array}{ll}M_{11} & M_{12} \\ M_{21} & M_{22}\end{array}\right):=V^{T}\left\{M(0)-\tilde{U} U^{T}\right\} V$.

The next $n$ equations of (4.1) are given by

$$
\Delta^{T}(n) \tilde{x}_{n}+\{M(n)-\lambda I\} \tilde{x}_{2 n}+\Delta(2 n) \tilde{x}_{3 n}=0,
$$

where $\Delta^{T}(n) \tilde{x}_{n}=\widetilde{\Delta}^{T} \tilde{\widetilde{x}}_{n}=\left(\tilde{\Delta}_{21} \tilde{\Delta}_{22}\right)^{T} x_{2}(n)$ by $(\mathrm{C} 2)$, because $x_{1}(n)=0$ by $(5.18)$. Hence, the next $n$ rows from $n-r_{b}+1, \ldots, 2 n-r_{b}$ of our matrix under construction have to be defined by

$$
\left(\left(\begin{array}{c}
\widetilde{\Delta}_{21}^{T} \\
\widetilde{\Delta}_{22}^{T}
\end{array}\right) M(n) \Delta(2 n) \quad 0 \cdots 0\right) .
$$

This completes the construction concerning the boundary conditions at the beginning. Thus, possible eigenvectors have to be of the form $\left(x_{2}^{T}(n), y_{n+1}, \ldots\right)$, where the boundary conditions are satisfied by putting $x_{1}(n)=0$ and defining $x_{2}(0)$ and $x_{1}(0)$ by (5.18) and (5.22), respectively.

\subsection{Boundary Conditions at the End}

We proceed similarly as in the previous subsection. Therefore, we can skip some details. We shall use for convenience the same notation for auxiliary matrices or vectors here, but of course, with a different meaning. Observe that the situation is nevertheless not symmetric (see the concluding remarks (ii) below).

We consider the boundary conditions (4.3) or (4.6) at the end by assuming (4.4) and (4.7), so that $T_{2}(N+1)$ and $\Delta(N+1)$ are invertible. Let $\tilde{X}, \tilde{U}$ be real $n \times n$-matrices such that (see (4.6))

$$
\tilde{X}^{T} \tilde{U}=\tilde{U}^{T} \tilde{X}, \quad \tilde{X}^{T} U-\tilde{U}^{T} X=I, \text { and } \tilde{X} \text { is invertible, }
$$

where $X:=\left(R_{e} \Delta^{-1}(N+1)\right)^{T}, U:=\left(R_{e}^{*}\right)^{T}$.

These matrices exist, because (5.5) holds by (4.4) and (4.6). Note that

$$
X=-T R_{N+1}^{T}
$$

by Proposition 3.2 and (4.6). Moreover, (5.7) holds as before. Hence, the boundary conditions (4.3) or (4.6) are equivalent with

$$
\tilde{X} U^{T} \tilde{x}_{N+1}+\tilde{X} X^{T} \Delta(N+1) \tilde{x}_{N+1+n}=0 .
$$


By the spectral theorem there exists an orthogonal matrix $\tilde{V}$ such that

$$
\tilde{V}^{T} \tilde{X} X^{T} \tilde{V}=\left(\begin{array}{ll}
D & 0 \\
0 & 0
\end{array}\right)
$$

where $D \in \mathbb{R}^{\left(n-r_{e}\right) \times\left(n-r_{e}\right)}$ is diagonal and invertible, so that

$$
r_{e}=n-\operatorname{rank} X=n-\operatorname{rank} R_{e}=n-\operatorname{rank} R_{N+1} .
$$

As before we use this block structure including the extreme cases $r_{e}=0$ and $r_{e}=n$. By QR-factorization there exists an orthogonal matrix $Q \in \mathbb{R}^{\left(n-r_{e}\right) \times\left(n-r_{e}\right)}$ such that $Q^{T} \Delta_{11}^{T}$ is upper triangular, where $\Delta(N+1-n) \tilde{V}:=\left(\begin{array}{c}\Delta_{11} \Delta_{12} \\ \Delta_{21} \Delta_{22}\end{array}\right)$ with the blockstructure above, that is, $\Delta_{22} \in \mathbb{R}^{r_{e} \times r_{e}}$. Then $V:=\tilde{V}\left(\begin{array}{cc}Q & 0 \\ 0 & I\end{array}\right)$ is orthogonal, and

$$
S:=V^{T}\left(-\tilde{X} X^{T}\right) V=\left(\begin{array}{cc}
S_{11} & 0 \\
0 & 0
\end{array}\right)
$$

where $S_{11}=-Q^{T} D Q$ is symmetric and invertible. Let

$$
R:=V^{T} \tilde{X} U^{T} V=\left(\begin{array}{ll}
R_{11} & R_{12} \\
R_{21} & R_{22}
\end{array}\right) .
$$

By (5.5) and (C5) we obtain that $R_{21}=0$ and that $R_{22}$ is invertible.

Altogether, we have constructed $V \in \mathbb{R}^{n \times n}$ such that

$$
S:=-V^{T} \tilde{X} X^{T} V=\left(\begin{array}{cc}
S_{11} & 0 \\
0 & 0
\end{array}\right)
$$

with a symmetric and invertible matrix $S_{11} \in \mathbb{R}^{\left(n-r_{e}\right) \times\left(n-r_{e}\right)}$, where $r_{e}=n-\operatorname{rank} X$,

$$
R:=V^{T} \tilde{X} U^{T} V=\left(\begin{array}{cc}
R_{11} & R_{12} \\
0 & R_{22}
\end{array}\right)
$$

with an invertible matrix $R_{22} \in \mathbb{R}^{r_{e} \times r_{e}}$ and so that $S_{11} R_{11}^{T}$ is symmetric, and

$$
\widetilde{\Delta}:=\Delta(N+1-n) V=\left(\begin{array}{cc}
\widetilde{\Delta}_{11} & \widetilde{\Delta}_{12} \\
\widetilde{\Delta}_{21} & \widetilde{\Delta}_{22}
\end{array}\right)
$$

so that $\widetilde{\Delta}_{11}^{T} \in \mathbb{R}^{\left(n-r_{e}\right) \times\left(n-r_{e}\right)}$ is upper triangular, and where $V$ is orthogonal. 
It follows from (C5) and (C6) that our boundary conditions (4.3) or (4.6) or (5.26) are equivalent with

$$
R V^{T} \tilde{x}_{N+1}+S V^{T} \Delta(N+1) \tilde{x}_{N+1+n}=0
$$

and therefore with

$$
\begin{aligned}
x_{2}(N+1)=0, \quad x_{1}(N+1+n)=-S_{11}^{-1} R_{11} x_{1}(N+1), \text { where } \\
\widetilde{\tilde{x}}_{N+1}:=V^{T} \tilde{x}_{N+1}=\left(\begin{array}{c}
x_{1}(N+1) \\
x_{2}(N+1)
\end{array}\right), \\
\tilde{\widetilde{x}}_{N+1+n}:=V^{T} \Delta(N+1) \tilde{x}_{N+1+n}=\left(\begin{array}{l}
x_{1}(N+1+n) \\
x_{2}(N+1+n)
\end{array}\right)
\end{aligned}
$$

with $x_{2}(N+1), x_{2}(N+1+n) \in \mathbb{R}^{r_{e}}, x_{1}(N+1)$, and $x_{2}(N+1+n)$ remaining free. We say that (5.34) is the boundary conditions (at the end) in normalized form.

Next, we consider the last $n$ equations of our difference equations (4.1) or (5.1), that is, by Lemma 2.1 and (5.2),

$$
v_{N+1-n}=\Delta^{T}(N+1-n) \tilde{x}_{N+1-n}+M(N+1-n) \tilde{x}_{N+1}+\Delta(N+1) \tilde{x}_{N+1+n}=\lambda \tilde{x}_{N+1} .
$$

Then, under (5.34) (i.e., the boundary conditions) this is equivalent with (using the notation of (5.34) and (C6))

$$
\tilde{\Delta}^{T} \tilde{x}_{N+1-n}+V^{T}\{M(N+1-n)-\lambda I\} V\left(\begin{array}{c}
x_{1}(N+1) \\
0
\end{array}\right)+\widetilde{\tilde{x}}_{N+1+n}=0,
$$

that is

$$
\begin{aligned}
& \left(\widetilde{\Delta}_{11}^{T}, \tilde{\Delta}_{21}^{T}\right) \tilde{x}_{N+1-n}+\left(M_{11}-\lambda I, M_{12}\right)\left(\begin{array}{c}
x_{1}(N+1) \\
0
\end{array}\right)+x_{1}(N+1+n)=0, \\
& \left(\tilde{\Delta}_{12}^{T}, \tilde{\Delta}_{22}^{T}\right) \tilde{x}_{N+1-n}+\left(M_{21}, M_{22}-\lambda I\right)\left(\begin{array}{c}
x_{1}(N+1) \\
0
\end{array}\right)+x_{2}(N+1+n)=0
\end{aligned}
$$

where $\left(\begin{array}{ll}M_{11} & M_{12} \\ M_{21} & M_{22}\end{array}\right):=V^{T} M(N+1-n) V$ is symmetric by Lemma 2.1. Hence, by (C6), equation (5.35) is equivalent with (under the boundary conditions (5.34))

$$
\begin{gathered}
x_{2}(N+1+n)=-\left(\widetilde{\Delta}_{12}^{T}, \tilde{\Delta}_{22}^{T}\right) \tilde{x}_{N+1-n}-M_{21} x_{1}(N+1), \\
\left(\widetilde{\Delta}_{11}^{T}, \widetilde{\Delta}_{21}^{T}\right) \tilde{x}_{N+1-n}+\{M-\lambda I\} x_{1}(N+1)=0, \quad \text { where } M:=M_{11}-S_{11}^{-1} R_{11} .
\end{gathered}
$$

Now, (5.38) defines $x_{2}(N+1+n)$ independently of $\lambda$, which was free by (5.34). Note that $M$ is symmetric, because $M(N+1-n)$ is symmetric by Lemma 2.1 and because $S_{11}^{-1} R_{11}$ is symmetric 
by (C6). Moreover, $\widetilde{\Delta}_{11}^{T}$ is upper triangular by (C6), so that (5.39) leads to bandwidth $2 n+1$ and symmetry. More precisely, we drop the last $n+r_{e}$ columns of $\mathcal{A}$, and the last $n$ rows are replaced by the following rows, which constitute the last $n-r_{e}$ rows of the symmetric, banded matrix under construction:

$$
\left(\begin{array}{lll}
0 \cdots 0 & \tilde{\Delta}_{11}^{T} \tilde{\Delta}_{21}^{T} & M
\end{array}\right), \quad \text { where } M:=M_{11}-S_{11}^{-1} R_{11} \in \mathbb{R}^{\left(n-r_{e}\right) \times\left(n-r_{e}\right)}
$$

and where $\left(\begin{array}{ll}M_{11} & M_{12} \\ M_{21} & M_{22}\end{array}\right):=V^{T} M(N+1-n) V$.

The last but one $n$ equations of (4.1) are given by

$$
\Delta^{T}(N+1-2 n) \tilde{x}_{N+1-2 n}+\{M(N+1-2 n)-\lambda I\} \tilde{x}_{N+1-n}+\Delta(N+1-n) \tilde{x}_{N+1}=0 .
$$

Note that for $2 n \leq N<3 n$ this overlaps with (5.24) of the previous subsection, and $\Delta(N+1-$ $2 n)$ may have been changed by the construction there. We use here this new $\Delta(N+1-2 n)$ from Section 5.1, but note that it is irrelevant here. By (C6) and (5.34) we have that

$$
\Delta(N+1-n) \tilde{x}(N+1)=\tilde{\Delta} \widetilde{\widetilde{x}}_{N+1}=\left(\begin{array}{c}
\widetilde{\Delta}_{11} \\
\tilde{\Delta}_{21}
\end{array}\right) x_{1}(N+1) .
$$

Hence, the $n$ rows before the $n-r_{e}$ rows of (C7) of our matrix under construction have to be defined by

$$
\left(\begin{array}{lll}
0 \cdots 0 & \Delta^{T}(N+1-2 n) & M(N+1-2 n)
\end{array}\left(\begin{array}{c}
\widetilde{\Delta}_{11} \\
\widetilde{\Delta}_{21}
\end{array}\right)\right)
$$

This completes the construction. Thus, possible eigenvectors must be of the form

$$
\left(x_{2}^{T}(n), y_{n+1}, \ldots, y_{N+1-n}, x_{1}^{T}(N+1)\right) \in \mathbb{R}^{N+1-r_{b}-r_{e}} .
$$

\section{Main Result and Concluding Remarks}

Altogether we have shown by the construction of Section 5 the following result.

Theorem 6.1. Assume (4.4) and (4.7). Then the construction of Section 5 transforms the SturmLiouville eigenvalue problem ( $(\mathcal{E})$ (given by (4.1) and (4.2)) of Section 4 into an equivalent algebraic eigenvalue problem for a real-symmetric, banded matrix with bandwidth $2 n+1$. This matrix is of size $\left(N+1-r_{b}-r_{e}\right) \times\left(N+1-r_{b}-r_{e}\right)$ with $r_{b}, r_{e} \in\{0,1, \ldots, n\}$ given by (5.10) and (5.28), and it is constructed from $A$ (defined by (5.2)) by (C1)-(C8).

\section{Concluding Remarks}

(i) By our theorem every discrete Sturm-Liouville eigenvalue problem $(\mathcal{\varepsilon})$ is equivalent with an algebraic eigenvalue problem for a banded, symmetric matrix under the assumptions (4.4) 
and (4.7). On the other hand, by [1, Remark 1(i)], such an algebraic eigenvalue problem is equivalent with a discrete Sturm-Liouville eigenvalue problem with Dirichlet boundary conditions. Moreover, if $r_{n}(k) \neq 0$ for all $k$, then our eigenvalue problem $(\varepsilon)$ can be written as an eigenvalue problem for a corresponding Hamiltonian system or symplectic system [14] according to Lemma 2.2. Note that it is quite natural to assume that the leading coefficient $r_{n}(k)$ never vanishes, because it is the case in most applications. But the main point is that the incorporation of our general boundary conditions into the difference equations by our construction leads in general to a problem where the leading coefficient may vanish for some $k^{\prime}$ s at the beginning and the end. To be more precise, our construction may cause that matrix elements $a_{k, k+n}$ become zero at the beginning and at the end, so that its equivalent Sturm-Liouville problem with Dirichlet boundary conditions will not satisfy $r_{n}(k) \neq 0$ for all $k$ anymore, because $a_{k, k+n}=(-1)^{n} r_{n}(k+n)$ by (2.3). Hence, it cannot be written as an eigenvalue problem for a linear Hamiltonian difference system, so that the corresponding recursion formulae (based on the Hamiltonian or associated Riccati difference system [4, 11]) cannot be applied for numerical purposes as in [4, Theorems $\mathrm{A}$ and 2]. Therefore, divisions by $r_{n}(k)$ must be avoided, which is done by the division-free algorithms presented in [4,5]. Hence, these division-free algorithms are crucial for our purposes.

(ii) Note that in contrast to the corresponding continuous Sturm-Liouville problems (or the corresponding Hamiltonian differential systems), there is no symmetry with respect to the endpoints in the discrete case. This is quite obvious by the difference equation (4.1) with forward differences. Therefore the treatment of the boundary conditions at the endpoints in the subsections above had to be done separately. Actually the results of this treatment are quite different as can be seen also from the next remark.

(iii) We discuss the extreme cases $r_{b}, r_{e}=0$ or $=n$ of our construction. It follows from (5.10) and (5.28) that $r_{b}=0$ if and only if $R_{b}^{*}$ is invertible, that is, $\tilde{x}_{0}=-\left(R_{b}^{*}\right)^{-1} R_{b} \tilde{x}_{n}$ by (4.5) (this includes Dirichlet conditions $\tilde{x}_{0}=0$ for $R_{b}=0$ ).

$r_{b}=n$ if and only if $R_{b}^{*}=0$, that is, $\tilde{x}_{n}=0$ by (4.5) (so that $\tilde{x}_{0}$ or $x_{0}$ is free). $r_{e}=$ 0 if and only if $R_{e}$ or $R_{N+1}$ is invertible, that is, $\tilde{x}_{N+1+n}=-R_{e}^{-1} R_{e}^{*} \tilde{x}_{N+1}$ by (4.6) or $u_{N+1}=$ $-R_{N+1}^{-1} R_{N+1}^{*} x_{N+1}$ by (4.3) (this includes natural boundary conditions $u_{N+1}=0$ for $R_{N+1}^{*}=0$ ). $r_{e}=n$ if and only if $R_{e}=0$, that is, $\tilde{x}_{N+1}=x_{N+1}=0$ (Dirichlet conditions).

Hence, our construction leads to the maximal size $(N+1) \times(N+1)$ (i.e., $\left.r_{b}=r_{e}=0\right)$ of the constructed matrix for Dirichlet conditions $\tilde{x}_{0}=x_{0}=0$ (more general for $\left.\tilde{x}_{0}=-\left(R_{b}^{*}\right)^{-1} R_{b} \tilde{x}_{n}\right)$ at the beginning and for natural boundary conditions (more general for $\tilde{x}_{N+1+n}=-R_{e}^{-1} R_{e}^{*} \tilde{x}_{N+1}$ ) at the end. The construction leads to the minimal size $(N+1-2 n) \times(N+1-2 n)$ for $\tilde{x}_{n}=0$ (or for free $\left.\tilde{x}_{0}\right)$ at the beginning and for Dirichlet conditions $\tilde{x}_{N+1}=x_{N+1}=0$ at the end.

\section{Acknowledgment}

This research is supported by Grant KR 1157/2-1 of DFG.

\section{References}

[1] W. Kratz, "Banded matrices and difference equations," Linear Algebra and Its Applications, vol. 337, no. $1-3$, pp. 1-20, 2001.

[2] J. H. Wilkinson, The Algebraic Eigenvalue Problem, Clarendon Press, Oxford, UK, 1965.

[3] W. Kratz, Quadratic Functionals in Variational Analysis and Control Theory, vol. 6 of Mathematical Topics, Akademie, Berlin, Germany, 1995.

[4] W. Kratz and M. Tentler, "Recursion formulae for the characteristic polynomial of symmetric banded matrices," Linear Algebra and Its Applications, vol. 428, no. 11-12, pp. 2482-2500, 2008. 
[5] M. Tentler, Rekursionsformeln zur Berechnung der charakteristischen Polynome von symmetrischen Bandmatrizen, Dissertation, Universität Ulm, Ulm, Germany, 2008.

[6] M. Bohner, O. Došlý, and W. Kratz, "Discrete Reid roundabout theorems," Dynamic Systems and Applications, vol. 8, no. 3-4, pp. 345-352, 1999.

[7] O. Došlý and W. Kratz, "Oscillation and spectral theory for symplectic difference systems with separated boundary conditions," to appear in Journal of Difference Equations and Applications.

[8] M. Bohner, O. Došly, and W. Kratz, "An oscillation theorem for discrete eigenvalue problems," The Rocky Mountain Journal of Mathematics, vol. 33, no. 4, pp. 1233-1260, 2003.

[9] M. Bohner and O. Došlý, "Disconjugacy and transformations for symplectic systems," The Rocky Mountain Journal of Mathematics, vol. 27, no. 3, pp. 707-743, 1997.

[10] M. Bohner, O. Došlý, and W. Kratz, "Inequalities and asymptotics for Riccati matrix difference operators," Journal of Mathematical Analysis and Applications, vol. 221, no. 1, pp. 262-286, 1998.

[11] C. D. Ahlbrandt and A. C. Peterson, Discrete Hamiltonian Systems: Difference Equations, Continued Fractions, and Riccati Equation, vol. 16 of Kluwer Texts in the Mathematical Sciences, Kluwer Academic Publishers, Dordrecht, The Netherlands, 1996.

[12] O. Došlý and W. Kratz, "Oscillation theorems for symplectic difference systems," Journal of Difference Equations and Applications, vol. 13, no. 7, pp. 585-605, 2007.

[13] G. Strang, Linear Algebra and Its Applications, Academic Press, New York, NY, USA, 1976.

[14] M. Bohner, O. Došly, and W. Kratz, "Sturmian and spectral theory for discrete symplectic systems," Transactions of the American Mathematical Society, vol. 361, no. 6, pp. 3109-3123, 2009. 